\title{
FAKE NEWS RESILIENCE THROUGH ONLINE GAMES? TENTATIVE FINDINGS FROM A RANDOMIZED CONTROLLED TRIAL IN HIGHER EDUCATION
}

\author{
Christoph Pimmer, Christoph Eisemann and Mateescu Magdalena \\ FHNW University of Applied Sciences and Arts Northwestern Switzerland \\ Peter Merian-Strasse 86, 4002 Basel - Switzerland
}

\begin{abstract}
Learners' cognitive abilities to assess the credibility of information in digital spaces are part of $21^{\text {st }}$ century skills. Emerging evidence suggests that gamification could be a suitable approach for learners to develop these skills independently of their educational level. This study examined two popular online fake news games in a higher education setting using a randomized controlled trial. 72 students were randomly assigned to one of two games. Their ability to classify news, i.e. to distinguish fake news from correct news, was tested before and after playing the game. The results from multiple regression analysis suggest that there was only a very modest increase in participants' news classification abilities in one game and no improvement in the other game. Contrary to some prior literature, these preliminary findings provide no evidence for the use of gamification in developing students' fake news resilience in higher education contexts and they call for more nuanced education and gamification approaches.
\end{abstract}

\section{KEYWORDS}

Fake News, Disinformation, Gamification, Serious Games, Fake News Resilience

\section{INTRODUCTION AND BACKGROUND}

False and fake news are a phenomenon which is deeply entrenched in today's information society. Fake news is connected to online media and social networks in particular, because on these platforms misinformation spreads remarkably farther, faster, deeper and more broadly than the truth (Vosoughi et al., 2018). The social and political consequences are potentially devastating. It has been suggested, for example, that false online stories can negatively impact political decision making in elections (Persily, 2017). In this light it is worrying that young people have little confidence in their ability to recognize fake news online (Notley \& Dezuanni, 2019). The most promising strategy in fighting fake news is conceived to be the education of users (Waldrop, 2017). However, in many cases the attempt to correct false beliefs had limited effects or was even counterproductive, particularly regarding polarizing topics, such as vaccination (e.g. Pluviano, Watt, Ragazzini, \& Della Sala, 2019).

A more pro-active way is thus to equip users with the methodological repertoire that permits them to detect fake news before misconceptions strike roots. A promising approach to achieving this is gamification. Games and gamified approaches are, according to a recent meta-reviews, more effective than many other forms of learning (e.g. Bai, Hew, \& Huang, 2020). In the field of fake news and media literacy education there is an increasing number of online games and gamified approaches available. However, the evidence for their effectiveness is thin. There are many games which have not been researched and the few published studies often concentrate on design and usability (e.g. Urban, Hewitt, \& Moore, 2018) or rely on self-reported measures. One of the few exceptions is the Bad News Game. A large-scale, pretest-post test study with $15^{\prime} 000$ participants provides initial evidence for the effectiveness of this game (Roozenbeek \& van der Linden, 2019) and a randomized controlled trial further affirmed these positive results (Basol, Roozenbeek, \& van der Linden, 2020). The main finding was that playing the online game "bad news" increased people's ability to detect disinformation mostly independently of their socio-demographic and political backgrounds - for example, regardless of their education (Roozenbeek \& van der Linden, 2019). 
The Bad News game is also interesting because it presents one of two predominant gamification approaches in fake news games: Users assume the role of fake news spreaders (Roozenbeek \& van der Linden, 2019). They learn about the mechanisms of deception by seeking to deceive others. The second main gamification approach is the classic, quiz-based concept of fake news detection, where users need to evaluate the credibility of news items, receiving instructions and feedback in this process.

Against this background, the present study explores the effects of two fake news online games on learners' news classification skills in a higher education context. The specific question is whether the games would improve learners' news classification abilities and if so, to what extent?

\section{MATERIALS AND METHODS}

We examined two games which were representative of the two main game fake news gaming approaches. For the fake news spreader approach, the above-described Bad News Game (the German version) was chosen. For the fake news detection approach, the online game Fakefinder from the German public broadcasting corporation Südwestrundfunk was selected. Fakefinder requires users to evaluate a series of news and provides instructions, feedback and background information in this process. The two online games cover similar content and they have also crucial gamification concepts in common, such as progress bars and badges (Bai et al., 2020). Their most notable difference is, as indicated, that players immerse themselves in different, antagonistic roles: either as fake news detectors or as fake news spreaders.

The research questions were addressed by carrying out a two-arm (1:1) repeated measurement randomized controlled trial $(n=72)$. Students from a bachelor degree program from a business school in Switzerland were randomized into two conditions (the two games). The randomization resulted in 19 male students and 17 female students in each of the two conditions. Before and after playing the game, a test was carried out which measured the participants' ability to correctly classify nine news items as fake news or correct news. The news presentation contained a screenshot and the link to the original news artifact that students could access in case they needed further background information. A score was calculated by crediting one point for each correctly classified news item.

\section{FINDINGS}

The descriptive statistics show a very small overall increase between the pre-test and the post-test across the two conditions. On average, participants increased their ability to detect fake news (i.e. their news classification score) from 6.29 points out of $9(70 \%)$ in the pre-test to $6.58(73 \%)$ in the post test. Table 2 shows the descriptive statistics per condition/game. It can be seen that the increase of the news classification score in the Fakefinder game condition was considerably more pronounced. (Table 1).

Table 1. Descriptive statistics in total and for each condition

\begin{tabular}{llllllll}
\hline News classification score & $\mathbf{n}$ & mean & sd & median & min & max & range \\
\hline Pre-test total & 72 & 6.29 & 1.76 & 6 & 2 & 9 & 7 \\
Post-test total & 72 & 6.58 & 1.57 & 7 & 2 & 9 & 7 \\
\hline Fake News spreader game: pre-test & 36 & 6.5 & 1.63 & 6.5 & 3 & 9 & 6 \\
Fake News spreader game: post-test & 36 & 6.56 & 1.59 & 6.5 & 2 & 9 & 7 \\
\hline Fake news detection game: pre-test & 36 & 6.08 & 1.89 & 6 & 2 & 9 & 7 \\
Fake news detection game: post-test & 36 & 6.61 & 1.57 & 7 & 3 & 9 & 6 \\
\hline
\end{tabular}


In the next step, the connections in the data were analysed through a mixed model regression analysis. To account for the repeated measures, the participants' ID was included as a random factor. We followed a model-contrasting approach using likelihood tests to compare the models (Pinheiro \& Bates, 2000). That is, various models were built in which the response variable news classification score was predicted by the group (the game), the interaction between time and group, and gender (see Table 2). The statistical analyses were performed in the computing environment $\mathrm{R}$. The mixed models were fitted with the nlme package (Pinheiro \& Bates, 2000), r-squared calculations drew on the MuMIn package (Nakagawa \& Schielzeth, 2013). Table 2 shows that Model 3 is superior to a random model and to all other models (LRatio Model $3=9.91$, $\mathrm{p}<.05)$. It has the highest predictive value, accounting for $28 \%$ of the variation.

Table 2. News classification score model and predictor variables

\begin{tabular}{llll} 
& Model 1 & Model 2 & Model 3 \\
\hline Condition & $-0.18(0.36)$ & $-0.42(0.40)$ & $-0.42(0.39)$ \\
Condition* Time (Fake News spreader game) & & $0.06(0.23)$ & $0.06(0.24)$ \\
Condition*Time (Fake news detection game) & & $0.53^{*}(0.23)$ & $0.53^{*}(0.24)$ \\
Gender (female) & & & $-0.75^{*}(0.35)$ \\
\hline AIC & 527.35 & 526.28 & 523.70 \\
BIC & 539.23 & 544.10 & 544.49 \\
Log Likelihood & -259.68 & -257.14 & -254.85 \\
Adj. R2 & .23 & .25 & .28 \\
Num. obs. & 144 & 144 & 144 \\
Num. groups: Number & 72 & 72 & 72 \\
\hline
\end{tabular}

${ }^{* * *} \mathrm{p}<0.001 ;{ }^{* *} \mathrm{p}<0.01 ;{ }^{*} \mathrm{p}<0.05$

The results show that there was a small but significant positive interaction effect between time and condition for the fake news detection game, but not for the fake news spreading game. In essence, the fake news detection game Fakefinder increased participants' news classification score very modestly whereas the fake news spreader game Bad News did not improve the score at all. Gender (i.e. being female) had a slightly negative effect on the news classification score.

\section{DISCUSSION}

The findings suggest that playing the fake news detection game amounted to a significant but very small increase in learners' fake news detection skills, whereas playing the fake news spreader game had no significant effects. These findings add to the current literature in that they relativize the impact of fake news games in higher education settings in general. They contrast with findings from Roozenbeek and van der Linden (2019) in particular, who reported that the fake news spreading game Bad News would increase people's ability to spot misinformation irrespective of their education level. The reasons for the limited impact in both games cannot be attributed to a ceiling effect in the response variable, as the learners' average pre-test score of $70 \%$ would have permitted room for improvement. These findings would not support the use of gamified interventions that draw on the design principles applied in the two games over other forms of learning and teaching. One way to achieve higher knowledge gains in a higher education context might be the integration of more advanced technical fake news detection strategies, such as image verification techniques (Katsaounidou, Vrysis, Kotsakis, Dimoulas, \& Veglis, 2019). Another option could be the integration of further gamification elements that increase the level of engagement and learning, such as competition. Or the use of constructionist learning where users truly create (and not just select) fake news. One example for this is the game studied by Clever et al. (2020), who, however, restricted this idea to the creation of headlines. 
Naturally, the study's findings are tentative in nature and need to be interpreted with respect to the methodological limitations. Firstly, the research involved a single site design and it is not clear to what extent the findings can be generalized across other, higher education contexts. Secondly, although we used a randomized between- group and within-group comparison design, the study involved a relatively small numbers of participants. And thirdly, the measurement approach of making users evaluate news, though quite common in the extant literature (e.g. Basol et al., 2020; Roozenbeek \& van der Linden, 2019), does not reflect the full concept of digital literacy which might have been gained through playing the games. For example, knowledge about the underlying dynamics of the media system was not covered. Finally, it needs to be acknowledged that the experiment did not reflect real world conditions. The students were primed by the instruction of detecting fake news, which is atypical of their informal, day-to-day news consumption. Future work might thus consider more subtle measurements of learners" "fake news" and media literacy skills.

\section{CONCLUSION}

Although preliminary in nature, the study suggests that the two games did not impact learners' news classification abilities in meaningful ways, which is in contrast to results from some previous studies. From a practical perspective, these findings call for more nuanced gaming approaches in a higher education context, as outlined.

\section{ACKNOWLEDGEMENT}

We would like to thank Christian Tanner, Programme Head Bachelor of Science in Betriebsökonomie Basel and Markus Freiburghaus, the Head of Education, both from the FHNW University of Applied Sciences and Arts Northwestern Switzerland School of Business, for their support of this study. We are also very grateful for the students who agreed to participate in this research.

\section{REFERENCES}

Bai, S., Hew, K. F., \& Huang, B. (2020). Is gamification "bullshit"? Evidence from a meta-analysis and synthesis of qualitative data in educational contexts. Educational Research Review, 100322.

Basol, M., Roozenbeek, J., \& van der Linden, S. (2020). Good News about Bad News: Gamified Inoculation Boosts Confidence and Cognitive Immunity Against Fake News. Journal of Cognition, 3(1).

Clever, L., Assenmacher, D., Müller, K., Seiler, M. V., Riehle, D. M., Preuss, M., \& Grimme, C. (2020). FakeYou! --A Gamified Approach for Building and Evaluating Resilience Against Fake News. arXiv preprint arXiv:2003.07595.

Katsaounidou, A., Vrysis, L., Kotsakis, R., Dimoulas, C., \& Veglis, A. (2019). MAthE the Game: A Serious Game for Education and Training in News Verification. Education Sciences, 9(2). doi:10.3390/educsci9020155

Nakagawa, S., \& Schielzeth, H. (2013). A general and simple method for obtaining R2 from generalized linear mixed-effects models. Methods in ecology and evolution, 4(2), 133-142.

Persily, N. (2017). The 2016 US Election: Can democracy survive the internet? Journal of democracy, 28(2), 63-76.

Pinheiro, J. C., \& Bates, D. M. (2000). Mixed-effects models in S and S-PLUS New York. NY: Springer-Verlag.

Pluviano, S., Watt, C., Ragazzini, G., \& Della Sala, S. (2019). Parents' beliefs in misinformation about vaccines are strengthened by pro-vaccine campaigns. Cognitive Processing, 20(3), 325-331. doi:10.1007/s10339-019-00919-w

Roozenbeek, J., \& van der Linden, S. (2019). Fake news game confers psychological resistance against online misinformation. Nature (Palgrave Communications), 5(1), 12.

Urban, A., Hewitt, C., \& Moore, J. (2018). Fake it to make it, media literacy, and persuasive design: Using the functional triad as a tool for investigating persuasive elements in a fake news simulator. Proceedings of the Association for Information Science and Technology, 55(1), 915-916.

Waldrop, M. M. (2017). News Feature: The genuine problem of fake news. Proceedings of the National Academy of Sciences, 114(48), 12631-12634. 